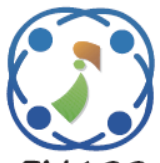

\title{
PSA-HD: Path Selection Algorithm based on Hamming Distance to Enhance the Link Stability in Mobile Ad-hoc Networks
}

\author{
Calduwel Newton Pitchai ${ }^{{ }^{*}} \quad$ Nismon Rio Robert $^{2}$ \\ ${ }^{1}$ Department of Computer Science, Government Arts College, \\ Ayyarmalai, Kulithalai-639 120, Karur-District, Tamil Nadu, India \\ ${ }^{2}$ Department of Computer Science, Bishop Heber College (Autonomous), \\ Tiruchirappalli-620 017, Tamil Nadu, India \\ * Corresponding author's Email: calduwel@yahoo.com
}

\begin{abstract}
Routing in Mobile Ad-hoc Networks (MANETs) is a very challenging task since, nodes are mobile and links are frequently created and broken. Traditional proactive routing algorithms initiate path discovery process from beginning after link failure occurrence. Generally, link failure occurs due to frequent topology change, fading, multiuser interference, etc., In MANETs link stability is the important criterion for link failure. Once the link meets with failure, it affects the overall performance of MANETs topology and it is very difficult to select optimum path as it leads to more transmission delay. The objective of this paper is to develop a mathematical model for Path Selection Algorithm based on Hamming Distance and delay parameters (PSA-HD). In this paper, link failure is identified, stable link is selected from the available links to reach the destination and it determines a stable path. The simulation result reveals that there is a decrease in average End-to-End delay and increase in Packet Delivery Ratio (PDR).
\end{abstract}

Keywords: Hamming distance, Delay, Link failure, Link stability and path selection.

\section{Introduction}

MANETs is one kind of wireless ad-hoc network and it is a self-configuring network of mobile routers connected by several wireless links to form a temporary network for sharing information among the users. Since it has the dynamic nature of ad-hoc networks, it leads to frequent changes in the path and unpredictability in changing the topology. These make the researchers active in routing area in MANETs because the mobile nodes can be setup at anytime, anywhere and they are free to move randomly. The links between the mobile nodes lead to frequent link breakages. Therefore, discovering and maintaining paths among the nodes form one of the major issues in MANETs.

There have been many routing metrics which are available. The most widely used metric is hop count. It is used in both the static and dynamic networks. The term "hop count" is used to represent the number of jumps (or hops) traversed by a packet between the source and destination. The minimum hop count between a specific source and destination is called the shortest path. Hop count is one of the best metrics for use in a static network in which, if there are multiple routing paths available, the path with the minimum hop count is selected. This is because with a shorter path, fewer resources will be consumed. There are many varieties of ad-hoc routing protocols to find an optimum path between the source and destination.

To detect the link break in topology, hamming distance concept is adopted with the proposed algorithm. Hamming distance is used to detect changes of bit positions between two binary values. Likewise, PSA-HD adopted this technique for MANETs to detect changes in path among multiple paths. Henceforth, routing table updated automatically by counting changes in the character position. Already a technique is proposed in the 
name of REfined Hamming DIStance (REHDIS) for stable path selection which can be found in [1]. As per PSA-HD, it yields the first priority to which the path has the hamming distance as 1 . As a result, the proposed PSA-HD algorithm selects an alternate link quickly in case of a link failure, it transfers the data quickly, reduces the End-to-End delay in turn and it improves in the Packet Delivery Ratio.

The rest of the section is structured as follows: Section 2 presents a brief review of various protocol and algorithms. Section 3 explores the proposed PSA-HD and highlights the mathematical model. Section 4 is the conclusion suggesting future work. Finally, references are listed.

\section{Related works}

This section presents some literature review of efficient route discovery and link stability based on the multicast routing protocol in MANETs. In the last few years many researchers proposed many mechanisms of multicast routing. An algorithm is developed [2] for Local Link Failure Recovery (LLFR) for ad hoc networks that establish recovery from link failures spontaneously at the point of link breakage. In such cases, a reliable link failure recovery is the main criterion that determined the performance of the network in terms of QoS. The LLFR is used in each node that collects RREP in the RREP Buffer Table (RBT) stack in the highest order of signal strength, which gets triggered during link failures. Once a link failure is detected, the intermediate node searches for an alternate path around the faulty area by choosing the first RREP that is stacked in the RBT and establishes a new route to the intended destination for sending the data packets without any delay in time.

Two route repair techniques are used to repair the broken link of the network. Either the routes can be repaired by re-establishing a new route starting from the source node or the routes can be repaired by the node that detects the link break along the path [3]. According to this approach, the different route repair values are defined on the basis of the active path of network and the link break value of the network for finding in which location the link break is occurring to initiate route repair mechanisms. The link is repaired locally for the performance enhancement of the basic ad-hoc on demand routing protocol [4]. Alternate path finding for the next-tonext node in case of link breakage is a better opinion of route management. These protocols are beaconless to improve bandwidth utilization. Control Overheads have been reduced by using $802.11 \mathrm{~b}$ protocol to maintain connectivity of the moving nodes.

The best routing path is found based on three factors such as nodes distance, power and node mobility [5]. Their objective was achieving sustainable connectivity that is an ultimate goal during MANETs construction. The QoS routing protocol is explained [6] based on Link Stability for MANETs to establish a stable path by calculating the mobility degree of a node relative to its neighbour.

QoS aware is addressed [7] of on demand routing in MANETs to incorporate link quality estimation based on mobility prediction of nodes to select the stable paths. Modified AODV routing protocol which discovers its route for sending data and video packets based on the stability of the path and mentioned some mechanisms for measuring the stability but the path stability seems to be good among all the mechanisms [8].

QoS requirements of real-time applications by using admission control scheme and a feedback scheme is recognized [9].The novel part of this QoS-aware routing protocol is the use of the approximate bandwidth estimation to react to network traffic. This approach implements these schemes by using two bandwidth estimation methods to find the residual bandwidth available at each node to support new streams. A scheme is estimated to get the information about total bandwidth for delay sensitive applications in MANETs [10]. It uses modified technique for bandwidth estimation and for route maintenance. This algorithm selects routes with least traffic and follows alternate route method for route maintenance.

Optimize a route for secure selection using Ant Colony Optimization that helps in finding the paths between two nodes in a network and selection of path can be changed dynamically according to the condition of the wireless network in case of network congestion [11]. This algorithm gives the best solution for the shortest path problem in Ad hoc networks. AODV protocol is enhanced for the problem of link failure, which in turn enhances the performance of the network and AODV protocol and selects the secure and shortest path in MANETs [12]. In this proposed approach, it considers the high vicinity between nodes and these nodes are used for data transmission.

Stability estimation is introduced to multicast routing based on relative stability metric and local stability metric for finding a stable path, in which, the already mentioned two decision factors (i.e., the relative stability metric and the local stability 
metric) help to estimate a concerned link's stability strength [13]. This developed protocol provides a flexible and feasible approach to choose a more efficient multicast tree in a reactive manner. Linear optimization based quality analysis of route and split the load according to route quality is proposed [14]. The optimization problem thus framed is by considering the network parameters such as Peak Signal Noise Ratio (PSNR) of images and path cost solving by which the quality of the routes is enhanced. Initially source node finds N node disjoint paths till destination. Probe packets from source to destination are sent to know the network condition. PSNR for each image through different paths are calculated.

Link quality is evaluated to improve routing by using Dynamic Source Routing protocol. Considering wireless link quality, a routing algorithm chooses better paths, if the host's list of recently seen requests, then discard route request packet totally [15]. When RREQ matches host's own address, then packet route record contains route by which request reached the host from RREQ initiator. Return a route copy to the initiator in a route reply packet. Otherwise, append host's address to RREQ packet's route record and re-broadcast RREQ. Multipath routing is improved based on link availability, neighboring nodes queuing delay, node mobility and bit error rate. The optimal path is selected using BAT meta-heuristic optimization [16]. The movement of a node from one location to another at a constant speed and direction is called epoch. The mobility of the nodes is comprised of multiple epochs which need not be the same.

PPCLSS means Predicted Probabilistic Coefficient Link Stability scheme based routing for MANETs [17]. Link loss is found based on a number of packets forwarded from a mobile node to the average dynamic distance between two mobile nodes with the mobility proportion. It considers the energy utilized for transmission of a packet and the average path lifetime. Link stability is estimated based on link connectivity changes, which can be performed on the network layer, without the need of peripheral devices or low layer data [18].

The route instability in AODV protocol is studied and suggested a solution for improvement [19]. This approach is used to reduce the route failure by storing the alternate route in the intermediate nodes. In this algorithm intermediate nodes are also involved in the route discovery process. As a result, it reduces the route establishment overhead as well as the time to find the reroute when a link failure occurs. Modified Termite Algorithms (MTA) is focused on development of an efficient routing algorithm for MANETs. The MTA developed by adopting efficient pheromone evaporation technique address to load balancing problems [20]. By including QoS, efficient route maintenance and local repair strategy by prediction of node failures, the MTA is expected to enhance the performance of the network in terms of throughput and reduction of End-to-End delay and routing overheads.

A light weight Proactive Source Routing Protocol (PSR) is proposed for data forwarding in MANETs. In PSR, each node maintained breadthfirst search spanning tree and that information is periodically exchanged among neighboring nodes for updating network topology information [21]. Thus, PSR allows a node to have full-path information to all other nodes in the network. In PSR, if link failure occurs at any node it starts finding the link failure from source node.

The disadvantages of the above mentioned algorithms and techniques were not capable of identifying the position of link failure. For this reason, PSA-HD is proposed and selects an alternate link from the failure node for its enhanced link stability in MANETs and reduction in average endto-end delay.

\section{PSA-HD: A mathematical model}

Let the number of nodes and links involved in wireless network be ' $n$ ' and ' $l$ ' respectively. A wireless network can be represented as a graph $G=$ $(V, E)$ where the nodes and links are represented as ' $V$ ' and ' $E$ ' respectively. Further, $V=\left\{v_{1}, v_{2}, \ldots, v_{n}\right\}$ and $E=\left\{e_{1}, e_{2}, \ldots, e_{l}\right\}$. Also $e_{i} \in E$ represents a link between $v_{i}$ and $v_{i-1}$. Without loss of generality, let $v_{l}=v_{S}$ and $v_{n}=v_{D}$ represents the source and destination of a wireless network. Thus (n-2)! alternate paths are possible between $v_{S}$ and $v_{D}$.

It is noted that link failure may happen at any time in any node.

The important assumption made in this paper is that path length from all intermediate nodes to the destination node is always the same. Assume that the link failure occurs at link $e_{i}$ where $e_{i}$ is positioned between the nodes $v_{i}$ and $v_{i+l}$. Let the failure node $v_{f}$ from $v_{S}$ is be maximum of $p$ position apart, where $p$ is defined as number of links between $v_{f}$ and $v_{D}$. Once the failure occurs at $v_{f}$. The number of possible path $(n-2-p)$ ! from $v_{f}$ to $v_{D}$ is not considered in forwarding the packet. Thus the total number of possible path is not considered in the entire wireless network and denoted as $T_{p}$ is 


$$
T_{p}=\sum_{r=p}^{n-p-2} \frac{(n-2-p) !}{(n-r) !}
$$

Once the failure occurs, the failure link $e_{i}$ where $e_{i} \epsilon$ $E\left(v_{i}, v_{i-1}\right)$ is identified and the path from $v_{i-1}$ to $v_{D}$ are completely removed and at the same time Route Error (RERR) is sent to the previous node $v_{i}$. From $v_{i}$ the alternate paths are found. Let it be $\mathrm{p}_{\mathrm{i}}$, where $i=1, \ldots, n_{A}, n_{A}<n$, where $n_{A}$ is number of alternate paths and the number of nodes involved in all $p_{i}$ are either the same or different. By keeping any one of the paths as stable say $p_{j}, p_{j} \subseteq p_{i}$ the rest of the paths are denoted as $p_{k}$, where $k=j+1, j+2, \ldots, n_{A}$ Hamming distance denoted as $d\left(h_{j}\right)=H\left(p_{j} \oplus p_{k}\right), j \neq k$ is found. To determine the optimal path $P S A H D_{\text {opt }}$ consider the following cases:

Case (i): if all $d(h)$ are distinct

After finding $d(h), P S A H D_{\text {opt }}$ is the one which has the least $d\left(h_{j}\right), j=p, p+1, \ldots, n$.

Case (ii): if some $d(h)$ are the same

Suppose two or more $d(h)$ are equal say $h_{\text {l, }}$ i.e., the number of links from $v_{i}$ to $v_{D}$ in all distinct paths are equal say $v_{e}$, the minimum delay $d_{\min }$ is considered in determining the $P S A H D_{\text {opt }}$, where $d_{\min }$ is calculated using

$$
\begin{aligned}
& \text { for each } p_{i}, i=1,2,3, \ldots, h_{l} \\
& d_{l i}=d\left(v_{s}, . ., v_{i}\right)+\sum_{i=1}^{v_{e}} d\left(v_{i}, v_{i+1}\right) \\
& P S A H D_{o p t}=\min \left\{d_{l i}\right\}
\end{aligned}
$$

Case (iii): if both $d(h)$ and $\mathrm{d}$ are equal

If two or more paths have both $d(h)$ and $\mathrm{d}$ as equal, the first path in the routing table is selected a PSAHD opt. $_{\text {. }}$

This section proves that the PSA-HD yields better results when compared with the existing protocols by formulating a mathematical model. There are five paths involved in the data transfer. They are $V_{S} \rightarrow V_{1} \rightarrow V_{2} \rightarrow V_{D}, \quad V_{S} \rightarrow V_{1} \rightarrow V_{4} \rightarrow V_{D}$, $\mathrm{V}_{\mathrm{S}} \rightarrow \mathrm{V}_{3} \rightarrow \mathrm{V}_{4} \rightarrow \mathrm{V}_{\mathrm{D}}, \quad \mathrm{V}_{\mathrm{S}} \rightarrow \mathrm{V}_{5} \rightarrow \mathrm{V}_{4} \rightarrow \mathrm{V}_{\mathrm{D}} \quad$ and $V_{S} \rightarrow V_{5} \rightarrow V_{6} \rightarrow V_{D}$. $P_{1}$ refers to the total number of links needed to transfer the data packets through the path $\left(\mathrm{V}_{\mathrm{S}} \rightarrow \mathrm{V}_{1} \rightarrow \mathrm{V}_{2} \rightarrow \mathrm{V}_{\mathrm{D}}\right)$. $\mathrm{P}_{2}$ refers to the total number of links needed to transfer the data packets through the path $\left(V_{S} \rightarrow V_{1} \rightarrow V_{4} \rightarrow V_{D}\right)$. $P_{3}$ refers to the total number of links needed to transfer the data packets through the $\left(V_{S} \rightarrow V_{3} \rightarrow V_{4} \rightarrow V_{D}\right)$. $P_{4}$ refers to the total number of links needed to transfer the data packets through the $\left(V_{S} \rightarrow V_{5} \rightarrow V_{4} \rightarrow V_{D}\right)$. $P_{5}$ refers to the total number of links needed to transfer the data
Table 1.Alternative Path(s) with Hamming Distance and Delay

\begin{tabular}{|c|c|c|c|}
\hline $\begin{array}{c}\text { No. of } \\
\text { Path(s) } \\
\text { Available }\end{array}$ & $\begin{array}{c}\text { Routing } \\
\text { Path }\end{array}$ & $\begin{array}{c}\text { Refined } \\
\text { Hamming } \\
\text { Distance }\end{array}$ & $\begin{array}{c}\text { Delay } \\
\text { (in } \\
\text { ms.) }\end{array}$ \\
\hline 1 & $\mathrm{VS}_{\mathrm{s}} \rightarrow \mathrm{V}_{1} \rightarrow \mathrm{V}_{4} \rightarrow \mathrm{V}_{\mathrm{D}}$ & 1 & 6 \\
\hline 2 & $\mathrm{VS} \rightarrow \mathrm{V}_{3} \rightarrow \mathrm{V}_{4} \rightarrow \mathrm{V}_{\mathrm{D}}$ & 2 & 7 \\
\hline 3 & $\mathrm{VS} \rightarrow \mathrm{V}_{5} \rightarrow \mathrm{V}_{4} \rightarrow \mathrm{V}_{\mathrm{D}}$ & 2 & 8 \\
\hline 4 & $\mathrm{VS} \rightarrow \mathrm{V}_{5} \rightarrow \mathrm{V}_{6} \rightarrow \mathrm{V}_{\mathrm{D}}$ & 2 & 5 \\
\hline
\end{tabular}

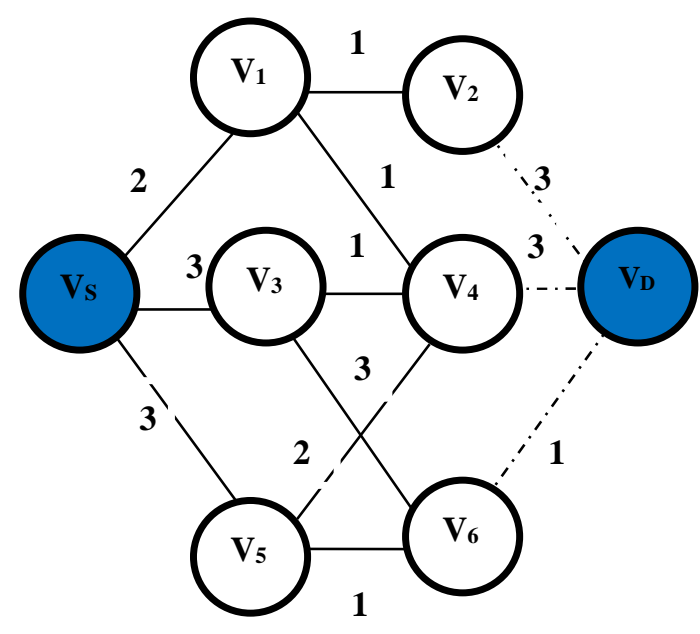

Figure.1 Network scenario 1

packets through the $\left(\mathrm{V}_{\mathrm{S}} \rightarrow \mathrm{V}_{5} \rightarrow \mathrm{V}_{6} \rightarrow \mathrm{V}_{\mathrm{D}}\right)$ as shown in Table 1. The PSA-HD identifies the number of links meeting with failure, it locally in turn reduces endto-end delay and increases PDR.

The PSA-HD algorithm is proposed to select the best optimum path among the multiple paths. It is described by considering the network scenario given in Fig.1. There are 8 nodes that are considered in the network scenario. They are $\mathrm{Vs}_{\mathrm{s}} \mathrm{V}_{1}, \mathrm{~V}_{2}, \mathrm{~V}_{3}, \mathrm{~V}_{4}, \mathrm{~V}_{5}$, $\mathrm{V}_{6}$ and $\mathrm{V}_{\mathrm{D}}$. The number on the edges denotes the delay in milli seconds. follows:

REfined Hamming DIstance is calculated as

$\begin{array}{llllll} & & 1 & 2 & 3 & 4 \\ & \text { Path1 } & \text { Vs } & \mathrm{V}_{1} & \mathrm{~V}_{2} & \mathrm{~V}_{\mathrm{D}} \\ \text { Path2 } & \mathrm{Vs} & \mathrm{V}_{1} & \mathrm{~V}_{4} & \mathrm{~V}_{\mathrm{D}} \\ \text { Refined Hamming } & = & & & 1 & \end{array}$

DIstance

Network Scenario 1:

The initial path is identified based on existing algorithms (i.e., $V_{s} \rightarrow V_{1} \rightarrow V_{2} \rightarrow V_{D}$ ). If $V_{2}$ leaves from the initial path as shown in Fig.2. PSA-HD algorithm identifies the alternative path from Table 1 based on refined hamming distance. Here, the alternative path, $V s \rightarrow V_{1} \rightarrow V_{4} \rightarrow V_{D}$ is selected as it has minimum distance, 1 . The PSA-HD gives the first priority to minimum refined hamming distance. 


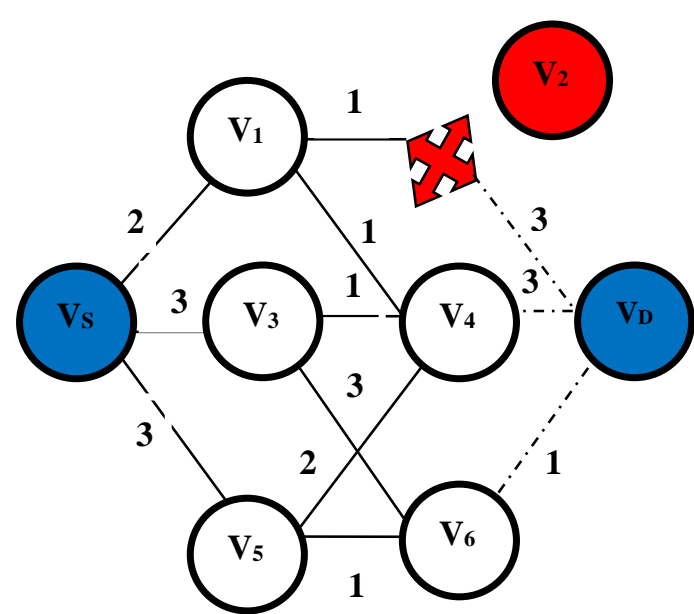

Figure. 2 Network scenario 2

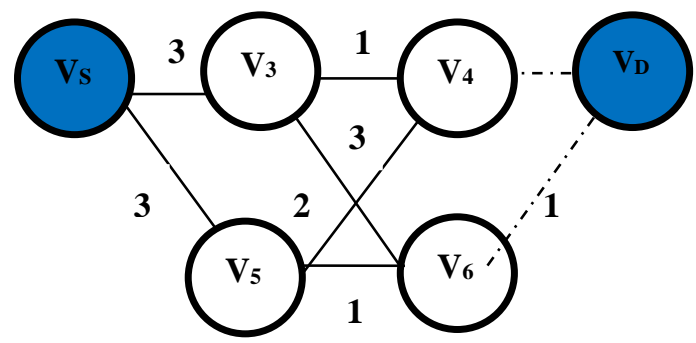

Figure. 3 Network scenario 3

In the above two paths, the character positions such as 1, 2 and 4 remain the same. But, in position 3 characters differ. There is a difference in one position out of the four positions. Hence, the refined hamming distance is 1 . Similarly, it is calculated for all other alternative paths.

\section{Network Scenario 2:}

If two alternative paths have the same distance, then it also considers delay. In another case, A leaves from the network as shown in Fig. 3. There is a need for new alternative path. The PSA-HD algorithm identifies the best optimum path from Table 2 . It identifies the alternative paths based on refined hamming distance. Here, there are two alternative paths available, $\quad \mathrm{Vs}_{\mathrm{S}} \rightarrow \mathrm{V}_{3} \rightarrow \mathrm{V}_{4} \rightarrow \mathrm{V}_{\mathrm{D}}$ and $\mathrm{V}_{\mathrm{S}} \rightarrow \mathrm{V}_{5} \rightarrow \mathrm{V}_{4} \rightarrow \mathrm{V}_{\mathrm{D}}$ as they have the minimum distance, 1 .

\section{Network Scenario 3:}

In this case, two paths have the same distance as shown in Fig.3. Hence, the best optimum path is selected based on the delay parameter. Here, the alternative path, $V_{s} \rightarrow V_{3} \rightarrow V_{4} \rightarrow V_{D}$ is selected as it has the minimum distance and delay compared to all other path. If the value of distance and delay are equal, then the best optimum path is selected from the alternative table which is calculated first as shown in Table 2.
Table 2.Alternative path(s) with Hamming distance and delay

\begin{tabular}{|c|c|c|c|}
\hline $\begin{array}{c}\text { No. of } \\
\text { Path (s) } \\
\text { Available }\end{array}$ & $\begin{array}{c}\text { Routing } \\
\text { Path }\end{array}$ & $\begin{array}{c}\text { Refined } \\
\text { Hamming } \\
\text { Distance }\end{array}$ & $\begin{array}{c}\text { Delay } \\
\text { (in } \\
\text { ms.) }\end{array}$ \\
\hline 1 & $\mathrm{~V}_{S} \rightarrow \mathrm{V}_{3} \rightarrow \mathrm{V}_{4} \rightarrow \mathrm{V}_{\mathrm{D}}$ & 1 & 7 \\
\hline 2 & $\mathrm{~V}_{S} \rightarrow \mathrm{V}_{3} \rightarrow \mathrm{V}_{6} \rightarrow \mathrm{V}_{\mathrm{D}}$ & 2 & 7 \\
\hline 3 & $\mathrm{~V}_{S} \rightarrow \mathrm{V}_{5} \rightarrow \mathrm{V}_{4} \rightarrow \mathrm{V}_{\mathrm{D}}$ & 1 & 8 \\
\hline 4 & $\mathrm{~V}_{\mathrm{S}} \rightarrow \mathrm{V}_{5} \rightarrow \mathrm{V}_{6} \rightarrow \mathrm{V}_{\mathrm{D}}$ & 2 & 5 \\
\hline
\end{tabular}

\section{Simulation results and analysis}

\section{- Simulation parameters}

In order to validate the working performance of the proposed algorithm PSA-HD with the existing Proactive Source Routing (PSR) and Predicted Probabilistic Coefficient Link Stability Scheme (PPCLSS) Table 3 shows the parameters and values used for the simulations.

\section{- Performance metrics}

The comparison has been done with the existing PSR and PPCLSS protocols with our proposed PSAHD. The following performance metrics are used to evaluate the performance of the PSA-HD.

\section{- Packet Delivery Ratio (PDR)}

It defines that Total number of packets received at destination divided by number of packets sent by source. It is used to measure the link stability of the discovered path.

\section{- Average end-to-end delay}

The End-to-End delay is referred to as the total time is taken for transmitting the data packets from source to destination.

Table 3. Simulation parameters

\begin{tabular}{|c|c|}
\hline Parameters & Values \\
\hline Number of Nodes & 20 \\
\hline Data Rate & $250 \mathrm{kbps}$ \\
\hline Network Size & $500 X 500 \mathrm{~m}^{2}$ \\
\hline MAC Protocol & IEEE 802.11 \\
\hline Simulation Time & 100 Seconds \\
\hline Traffic Agent & CBR \\
\hline Packet Size & $512 \mathrm{~kb}$ \\
\hline Mobility Model & Random Way Point Model \\
\hline Speed & $5,15,25,35$ and $50 \mathrm{~m} / \mathrm{s}$ \\
\hline Pause Time & 5 Seconds \\
\hline Antenna Type & Omni Antenna \\
\hline Radio Range & $250 \mathrm{~m}$ \\
\hline Routing Protocols & PSR, PPCLSS \\
\hline
\end{tabular}


Table 4. Computational time calculation

\begin{tabular}{|c|c|c|c|c|c|c|c|}
\hline \multicolumn{2}{|c|}{$\begin{array}{c}\text { Mobility } \\
\text { of Nodes }\left(\mathrm{m}_{\mathrm{i}}\right)\end{array}$} & 5 & 15 & 25 & 35 & 50 & $\frac{\boldsymbol{m}_{\boldsymbol{i}} \cdot \boldsymbol{d}_{\boldsymbol{i}}}{\boldsymbol{m}_{\boldsymbol{i}}}$ \\
\hline \multirow{2}{*}{$\begin{array}{c}\text { Delay }\left(\mathrm{d}_{\mathrm{i}}\right) \\
\text { (in ms) }\end{array}$} & PSR & 0.346 & 1.226 & 2.112 & 3.008 & 4.786 & 3.211 \\
\cline { 2 - 9 } & PPCLSS & 0.223 & 1.021 & 1.176 & 2.878 & 3.666 & 2.537 \\
\cline { 2 - 9 } & PSA-HD & 0.113 & 0.911 & 1.108 & 1.978 & 2.567 & 1.842 \\
\hline
\end{tabular}

Table 5. Packet delivery calculation

\begin{tabular}{|c|c|c|c|c|c|c|c|}
\hline \multicolumn{2}{|c|}{$\begin{array}{c}\text { Mobility } \\
\text { of Nodes }\left(\mathrm{m}_{\mathrm{i}}\right)\end{array}$} & 5 & 15 & 25 & 35 & 50 & $\frac{\boldsymbol{m}_{\boldsymbol{i}} \cdot \boldsymbol{d}_{\boldsymbol{i}}}{\boldsymbol{m}_{\boldsymbol{i}}}$ \\
\hline \multirow{2}{*}{$\begin{array}{c}\text { No. of } \\
\text { packets }\left(\mathrm{p}_{\mathrm{i}}\right)\end{array}$} & PSR & 91.6 & 93.12 & 94.15 & 95.18 & 96.2 & 94.97 \\
\cline { 2 - 9 }$(\mathrm{in} \%)$ & PPCLSS & 92.09 & 93.78 & 94.66 & 96.96 & 98.72 & 96.64 \\
\cline { 2 - 9 }
\end{tabular}

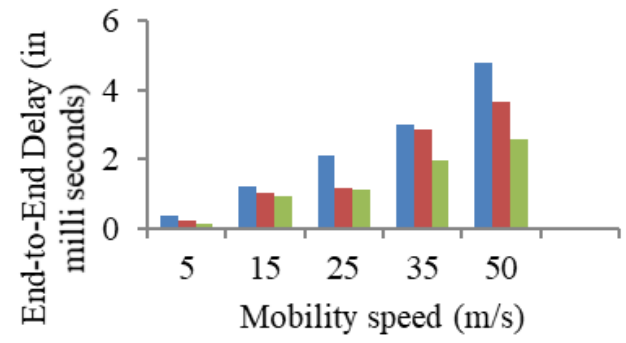

घSR

- PPCLSS

PSA-HD

Figure.4 End-to-end delay among PSR, PPCLSS, and PSA-HD

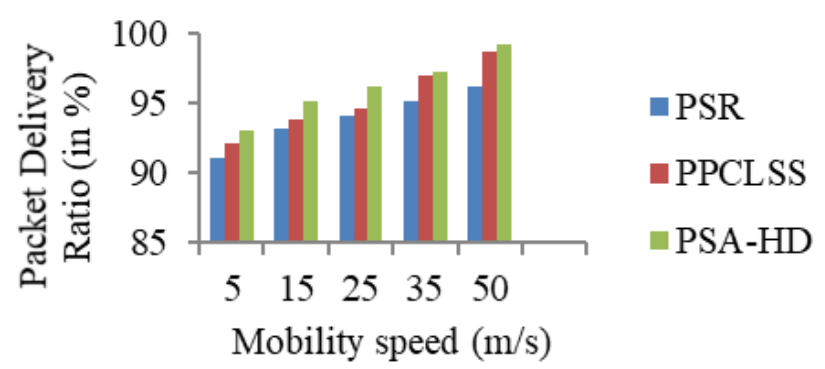

Figure.5 Packet delivery ratio among PSR, PPCLSS, and PSA-HD

\section{- Performance analysis}

In the experiment, the node speed varying from $5,15,25,35$ and $50 \mathrm{~m} / \mathrm{s}$ by keeping the nodes as constant rate and data rate as $250 \mathrm{~kb}$. Fig. 4 shows the comparative performance of PSR, PPCLSS and PSA-HD for different mobility speeds. The performance of the routing protocols is degraded when the mobility speed increases and when the mobility is increased from 5,15,25,35 to $50 \mathrm{~m} / \mathrm{s}$ the link failure increases, resulting in more route setup delay.

In PPCLSS [17] and PSR [21], if link failure occurs at any node it starts finding the link failure from source node. The proposed PSA-HD incurs less delay than the existing PSR and PPCLSS protocols as shown in Table 4.

The End-to-End delay increases gradually as the speed increases as depicted in Fig.4. Graphical representation shows the proposed PSA-HD outperforming PSR and PPCLSS with respect to delay.

The $\mathrm{X}$ axis represents the mobility speed and $\mathrm{Y}$ axis represents the average end-to-end delay in milli seconds.
Delay of existing algorithm

$$
\mathrm{d}_{\mathrm{li}}=\mathrm{d}\left(\mathrm{v}_{\mathrm{S}}, \ldots, \mathrm{v}_{\mathrm{i}}\right)+\sum_{i=1}^{v_{e}} \mathrm{~d}\left(\mathrm{v}_{\mathrm{i}}, \mathrm{v}_{\mathrm{i}+1}\right)
$$

Delay of proposed algorithm

$$
\mathrm{d}_{\mathrm{li}}=\sum_{i=1}^{v_{e}} \mathrm{~d}\left(\mathrm{v}_{\mathrm{i}}, \mathrm{v}_{\mathrm{i}+1}\right)
$$

Table 5 shows the PDR calculation among PSR, PPCLSS and PSA-HD in which, PSR and PPCLSS improve PDR to $94.97 \%$ and $96.64 \%$ respectively, whereas, PSA-HD improves to $97.36 \%$.

From the performance analysis, the results are concluded that PSA-HD shows superiority over PSR and PPCLSS. The proposed PSA-HD minimizes the delay and maximizes the PDR as depicted in Fig. 5.

\section{Conclusion and future work}

In this paper, PSA-HD algorithm has been proposed to address some of the issues like link failure and minimization of delay. The proposed PSA-HD identifies the failure node position in the path and selects an alternate link from the failure node. The mathematical model and simulation results prove that the proposed PSA-HD significantly reduces the end-to-end delay. The comparison clearly depicts that PSA-HD yields better results than PSR and PPCLSS. It identifies the path which has a minimum number of hamming distance and minimum delay compared to PSR and PPCLSS as it identify and repair the failure link locally. Even, if the mobility of the node is increased, PSA-HD gives better results. PDR calculation is done among PSR, PPCLSS and PSAHD in which, PSR and PPCLSS improve PDR to 94.97\% and $96.64 \%$ respectively, whereas, PSA-HD improves to $97.36 \%$. In general, the proposed PSAHD enhances the link stability in Mobile Ad-hoc Networks. In the future work, this proposed 
algorithm will be analyzed with Link Expiration Time (LET) where the links are survived for a longer duration of time along with minimum connected neighbors.

\section{Acknowledgments}

This work is financially supported by the University Grants Commission, Maulana Azad National Fellowship New Delhi, India.

\section{References}

[1] C. Newton and N. Rio, "REHDIS: Refined Hamming Distance technique to minimize data transfer delay in Mobile Ad-hoc Networks", In: Proc. of $11^{\text {th }}$ International Conf. on Intelligent Systems and Control ISCO, Coimbatore, India, pp.495-500, 2017.

[2] J. Jeni, V. Julie, and M. Bose, "An Enhanced Route Failure Recovery Model for Mobile Ad Hoc Networks", Journal of Computer Science, Vol.10, No. 8, pp. 1561-1568, 2014.

[3] S. Patle and P. Kumar, "Decision Making Approach to Prefer Route Repair Technique in AODV Routing Protocol of MANET", International Journal of Research in Engineering and Technology, Vol. 4, No. 2, pp. 9-16, 2015.

[4] J. Jain, R. Gupta, and T. Bandhopadhyaya, "Performance analysis of proposed local link repair schemes for ad hoc on demand distance vector", The Institution of Engineering and Technology, Vol. 3, No. 2, pp. 129-136, 2014.

[5] A. Yasin, S. Jabareen, and I. Al suqi, "Enhancing the connectivity of Mobile Ad- hoc Networks by considering the Power, mobility, and activity of nodes", International Journal of Computer Science, Vol. 11, No. 2, pp. 140-145, 2014.

[6] A. Moussaoui, F. Semchedine, and A. Boukerram, "A link-state QoS routing protocol based on link stability for Mobile Ad-hoc Networks", Journal of Network and Computer Applications, Vol.39, pp. 117-125, 2014.

[7] R. Patil, M. Rani.R, and R. Bainoor, "Link Stability Based On QoS Aware On Demand Routing in Mobile Ad Hoc Networks", IOSR Journal of Computer Engineering, Vol. 5, No. 3, pp. 52-57, 2012.

[8] T. Rajesh, M. Reddy, and K. Babu, "Relational Permanence Routing Protocol under Video Transmission for MANET", International Journal of Computer Science \& Communication Networks, Vol. 1, No. 3, pp. 212-217, 2011.
[9] E. Ravindra and P. Agraharkar, "QoS-Aware Routing Based on Bandwidth Estimation for Mobile Ad Hoc Networks", International Journal of Advance Electrical and Electronics Engineering, Vol. 3, No. 2, pp. 11-16, 2014.

[10] Surjeet, A. Parkash and R. Tripathi, "QoS Bandwidth Estimation Scheme for Delay Sensitive Applications in MANET", Communications and Network, Vol. 5, No. 1, pp. 1-8, 2013.

[11] S. Maheshwari and M. Bhardwaj, "Secure Route Selection in MANET Using Ant Colony Optimization", American Journal of Networks and Communications, Vol. 4, No. 3-1, pp.54-56, 2015.

[12] G.S. Hundal, R. Bedi, and S.K. Gupta, "Enhancement in AODV protocol to select the secure and shortest path in Mobile Ad hoc Network", International Journal of Computer Science and Information Technologies, Vol. 5, No. 5, pp. 6543-6546, 2014.

[13] H. Xia, S. Xia, J. Yu, Z. Jia, H. Edwin, and M. Sha, "Applying link stability estimation mechanism to multicast routing in MANET", Journal of Systems Architecture, Vol. 60, pp. 467- 480, 2013.

[14] V. Gatate and S. Terdal, "Enhancing Route Quality in MANET using Linear Optimization", International Journal of Advanced Research in Computer Engineering \& Technology, Vol. 1, No. 7, pp. 75-79, 2012.

[15] N. Prasath and P. Sengottuvelan, "Evaluation of Link Quality for Routing in DSR", Journal of Theoretical and Applied Information Technology, Vol. 59, No.1, pp. 183-188, 2014.

[16] R. Prabha and N. Ramaraj, "An improved multipath MANET routing using link estimation and Swarm Intelligence", Journal on Wireless Communications and Networking, pp. 1-9, 2015.

[17] D. Priya and P. Priyanka, "Probabilistic Prediction Coefficient Link Stability Scheme based Routing in MANET", International Journal of Computer Science \& Engineering Technology, Vol. 6, No. 4, pp. 246-256, 2015.

[18] Q. Song, Z. Ning, S. Wang, and A. Jamalipour," Link Stability Estimation Based on Link Connectivity Changes in Mobile Ad-hoc Networks", Journal of Network and Computer Applications, Vol. 35, No. 6, pp.1-26, 2012.

[19] K.G. Preetha, A. Unnikrishnan and P. Jacob, "An Effective Path Protection Method to Attain the Route Stability in MANET", International Journal of Advanced Research in Computer 
and Communication Engineering, Vol. 2, No. 6, pp. 2343-2348, 2013.

[20] G.S. Sharvani, A.G. Ananth, and T.M. Rangaswamy, "Efficient Stagnation Avoidance for MANETS with Local Repair Strategy Using Ant Colony Optimization", International Journal of Distributed and Parallel Systems, Vol.3, No.5, pp. 123-137, 2012.

[21] Z. Wang, Y. Chen, and C. Li, "PSR: A light weight Proactive Source Routing Protocol for Mobile Ad Hoc Networks", IEEE Transactions on Vehicular Technology, Vol. 63, No. 2, pp. 859-868, 2014. 